\title{
Modulation of halogen, chalcogen and pnictogen bonds by $\sigma$-hole tuning
}

\author{
J Fanfrlík \\ Institute of Organic Chemistry and Biochemistry of the Czech Academy of Sciences, Prague, Czech Republic
}

fanfrlik@uochb.cas.cz

Propertios of halogen (X), chalcogen (E) and pnictogen (Pn) bonds can be modulated by changing (i) the nature of the X, E and Pn, (ii) the chemical environment of the X, E and Pn, and (iii) properties of the electron donor. Apart from small molecular complexes [1], this has been demonstrated in protein-ligand complexes, e.g. on a series of aldose reductase inhibitors [2]. The counterintuitive ability of heteroboranes to form strong $\sigma$-hole interactions was found and attributed to the multicenter bonding [3]. It breaks the classical electronegativity concept and results highly positive $\sigma$-holes on heterovetices that are incorporated into the skeleton via multicenter type of bonding [3]. X, E and Pn elements in neutral heteroboranes can thus have highly positive $\sigma$-holes that are responsible for strong $\sigma$-hole interactions. The E $\cdots \pi$ [4], X $\cdots \pi$ [5], Pn $\cdots \pi$ [6] and $\mathrm{Pn} \cdots \mathrm{H}$-B [7] types of $\sigma$-hole interactions of heteroboranes have been observed in the corresponding crystal packings. $\sigma$-Hole interactions can be used for designing protein-ligand interactions as well as for crystal engineering.

(a)

(b)

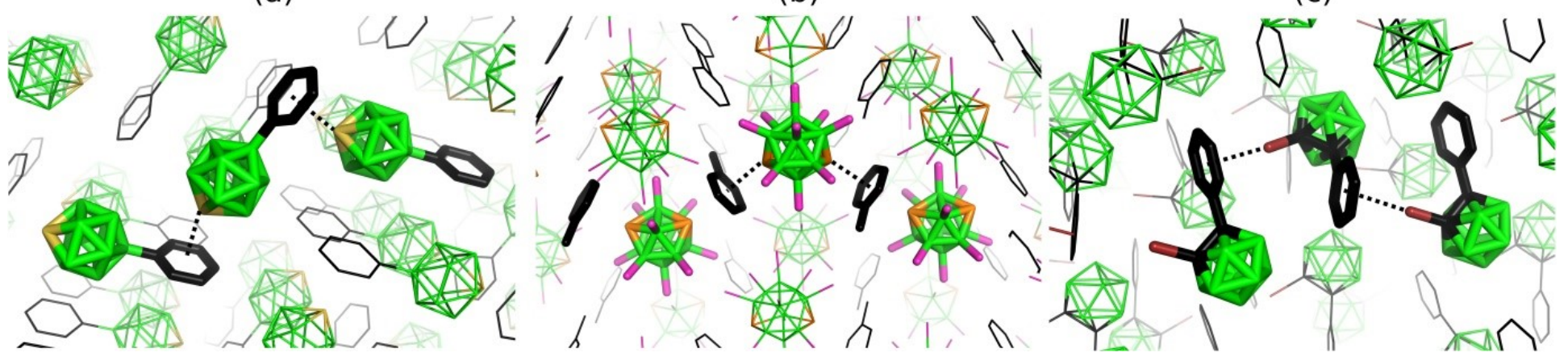

Figure 1. Chalcogen (a), pnictogen (b) and halogen (c) bonding found in heteroborane crystal structures [4-6]. Color coding: black carbon, yellow - sulfur, orange - phosphorus, pink - boron, dark red - bromine, magenta - chlorine. Hydrogen atom not shown.

[1] Riley, K. E., Murray, J. S., Fanfrlík, J., Řezáč, J., Solá, R. J., Concha, M. C., Ramos, F. M., \& Politzer, P. (2011) J. Mol. Model. 17, 3309.

[2] Fanfrlík, J., Kolář, M., Kamlar, M., Hurny, D., Ruiz, F. X., Cousido-Siah, A., Mitschler, A., Řezáč, J., Munusamy, E., Lepšík, M., Matějíček, P., Velselý, J., Podjarny, A., \& Hobza, P. (2013) ACS Chem. Biol. 8, 2484.

[3] Melichar, P., Hnyk, D., \& Fanfrlík, J. (2018) Phys. Chem. Chem. Phys. 20, 4666.

[4] Fanfrlík, J., Prada, A., Padělková, Z., Pecina, A., Macháček, J., Lepšík, M., Holub, J., Růžička, A., Hnyk, D., \& Hobza, P. (2014) Angew. Chem. Int. Ed. 58, 10139.

[5] Fanrlík, J., Holub, J., Růžičková, Z., Řezáč, J., Lade, P. D., Wand, D. A., Hnyk, D., Růžička, A., \& Hobza, P. (2016) ChemPhysChem $17,3373$.

[6] Fanfrlík, J. \& Hnyk, D. (2018) Crystals, 8, 390.

[7] Holub, J., Melichar, P., Růžičková, Z., Vrána, A., Wann, D. A., Fanfrlík, J., Hnyk, D., \& Růžička, A. (2017) Dalton Trans. 46, 137140.

Keywords: sigma-hole; computations; electrostatic potential

This work was supported by the European Regional Development Fund; OP RDE; Project: "Chemical biology for drugging undruggable targets (ChemBioDrug)" (No. CZ.02.1.01/0.0/0.0/16_019/0000729). 